\title{
Pronostic des implants zygomatiques dans la réhabilitation du sujet partiellement ou totalement édenté maxillaire : étude rétrospective observationnelle
}

\author{
R. Lan (Marseille), R. Castro (Marseille), L. Ammar (Marseille), P. Roche-Poggi \\ (Marseille), P. Tavitian (Marseille), 0. Richard (Marseille)
}

Introduction : Les implants zygomatiques semblent être une bonne alternative dans les cas de patients opérés de maxillectomies mais aussi pour les patients présentant une atrophie osseuse maxillaire sévère avec demande de réhabilitation prothétique sans avoir recours à des étapes de chirurgies pré-implantaires lourdes et chronophages. L'objectif principal de ce travail est d'évaluer le taux de succès et de survie chirurgical et prothétique des réhabilitations prothétiques zygo-implanto-portés avec un recul entre 1 et 10 ans. Méthodes et résultats : Une étude rétrospective observationnelle a été menée au pavillon d'odontologie de Marseille avec accord du Comité de Protection des Personnes. Trente patients opérés par le même praticien ayant bénéficiés d'implants zygomatiques dans des cas d'atrophie osseuse maxillaire sévère avec édentements complets et réhabilité par une prothèse supra-implantaire fixe soutenue par 1,2 ou 4 implants zygomatiques et entre 2 et 6 implants dentaires «standards » ont été inclus. Une analyse radiographique comparative a été réalisée au moyen des radiographies panoramiques et tomographie volumique à faisceau conique post-opératoires immédiats et au moment de l'inclusion afin d'analyser la qualité de l'ostéointégration. Des radiographies rétro-alvéolaire de chaque implant ont également été effectué afin d'analyser le niveau de résorption osseuse péri-implantaire. Le niveau d'inflammation gingivale, et la gestion des complications au niveau de la sphère orale et sinusienne ont été recueillis dans l'objectif d'évaluer le taux de succès et de survie tant au niveau chirurgical que prothétique des réhabilitations prothétiques zygo-implanto-portés. Bien que des cas de péri-implantites, de résorption osseuse ou de défaut d'axe empêchant la mise en fonction des implants immédiates ou différés ont été retrouvés, le taux de réussite chirurgical retrouvé pour les implants zygomatiques est équivalent à celui de l'implantologie standard et le taux de succès prothétique final a été assuré pour l'ensemble des cas retrouvés dans cette étude avec de très bon résultats esthétiques et fonctionnels à long terme. DIscussion et conclusion : A travers une sélection rigoureuse des cas, des indications adéquates et une connaissance de la technique chirurgicale mais aussi de ces complications, l'utilisation des implants zygomatiques associés à l'implantologie standard semble représenter un avantage certain dans la réhabilitation des maxillaires sévèrement résorbés, avec un cout similaire aux protocoles standards. La possibilité de mise en charge immédiate permet à la fois de minimiser le temps de latence entre la chirurgie et la réhabilitation orale et d'offrir une réinsertion sociale et professionnelle rapide augmentant le confort et la qualité de vie des patients. Une coopération étroite entre le clinicien en charge de la partie chirurgicale et le praticien responsable de la phase prothétique est indispensable.

D’Agostino A et al. J Oral Maxillofac Surg. 2016; 74(8):1562-1573.

Wang F et al. Int J Oral Maxillofac Implants. 2015; 30(2):293-8.

Nocini PF et al. Oral Maxillofac Surg. 2016;20(3):303-308. 